\title{
Direct measurement of skeletal muscle fatigue in patients with chronic heart failure
}

Nigel P Buller, David Jones, Philip A Poole-Wilson

Abstract

Skeletal muscle function was measured as force production and fatigue in both the quadriceps (a large locomotive muscle) and adductor pollicis (a small intrinsic hand muscle) in five healthy volunteers, five patients with mild chronic heart failure, and five patients with severe chronic heart failure. The quadriceps of patients with chronic heart failure had a reduced muscle cross sectional area, a reduced maximum isometric force production, and an increased tendency to fatigue. Isometric force production and fatigue of the adductor pollicis, however, were not significantly different between the three groups under control conditions. But during circulatory occlusion fatigue in the adductor pollicis increased more in the patients with severe chronic heart failure. These differing findings in quadriceps and adductor pollicis suggest that skeletal muscle atrophy and reduced isometric force production are not a necessary consequence of chronic heart failure per se, because they were only present in the large locomotive muscle. The normal values for muscle fatigue observed in adductor pollicis in patients with chronic heart failure imply that skeletal muscle blood flow must increase normally during muscle activation when only a small muscle mass is used. These results are not compatible with the concept of a generalised impairment of normal vasodilatation within active skeletal muscle. In contrast, activation of a large muscle, such as quadriceps, results in the rapid onset of fatigue in patients with severe chronic heart failure. This fatigue may be related to the inability of the cardiovascular system to provide the required blood flow for the activation of a large muscle mass. The finding of a relatively greater increase in fatigue of adductor pollicis during circulatory occlusion in patients with severe chronic heart failure supports the hypothesis of an intrinsic abnormality of skeletal muscle in these patients.

Exertional fatigue is a major symptom of patients with chronic heart failure. ${ }^{1}$ The mechanisms underlying this symptom are complex, but it has been established that reduced skeletal muscle blood flow on exercise is an important factor. ${ }^{2}$ The relative contributions to the reduced skeletal muscle blood flow of a reduced cardiac output and impaired vasodilatation within the skeletal muscle vascular bed are still unresolved. ${ }^{3-5}$ There is also evidence from studies with magnetic resonance spectroscopy of an intrinsic intracellular abnormality of skeletal muscle biochemistry in chronic heart failure. ${ }^{67} \mathrm{~A}$ study of exercise involving a small muscle mass in patients with chronic heart failure showed abnormal muscle biochemistry despite normal skeletal muscle blood flow?

We report on the direct measurement of skeletal muscle function in both the quadriceps (a large locomotive muscle) and the adductor pollicis (a small intrinsic hand muscle). The purpose of this study was to answer three questions. First, does the direct measurement of skeletal muscle fatigue correlate with the severity of chronic heart failure? Second, is fatigue dependent on the size of the muscle mass activated? Third, are factors other than skeletal muscle blood flow important in the genesis of fatigue in patients with chronic heart failure?

\section{Patients and methods}

The protocol was approved by the hospital ethics committee and all subjects gave informed consent.

\section{Patients}

We studied 15 right handed men (table): five healthy volunteers, five patients with mild or moderate chronic heart failure $\left(\dot{\mathrm{VO}}_{2} \max 15-\right.$ $25 \mathrm{ml} / \mathrm{min} / \mathrm{kg}$ ), and five patients with severe chronic heart failure $\left(\dot{\mathrm{Vo}}_{2} \max <15 \mathrm{ml} / \mathrm{min} /\right.$ $\mathrm{kg}$ ). We excluded patients with diabetes, thyroid dysfunction, neurological disease, arthritis, or orthopaedic abnormality. The cause of chronic heart failure was ischaemic heart disease. The five patients with mild heart failure were all in sinus rhythm. None was taking digoxin and three were taking an angiotensin converting enzyme inhibitor. The mean dose of frusemide was $56 \mathrm{mg} /$ day. Of the five patients with severe heart failure one was in atrial fibrillation, two were taking digoxin, and all were taking an angiotensin converting enzyme inhibitor. The mean dose of frusemide was $112 \mathrm{mg} /$ day. All patients were ambulant and considered to be receiving optimal oral medication at the time of the study. \\ and Lung Institute, \\ London \\ The Rayne Institute, \\ Hospital Medical \\ Correspondence to \\ Dr Nigel P Buller, \\ Department of Cardiology,
}


Table Results in adductor pollicis and quadriceps

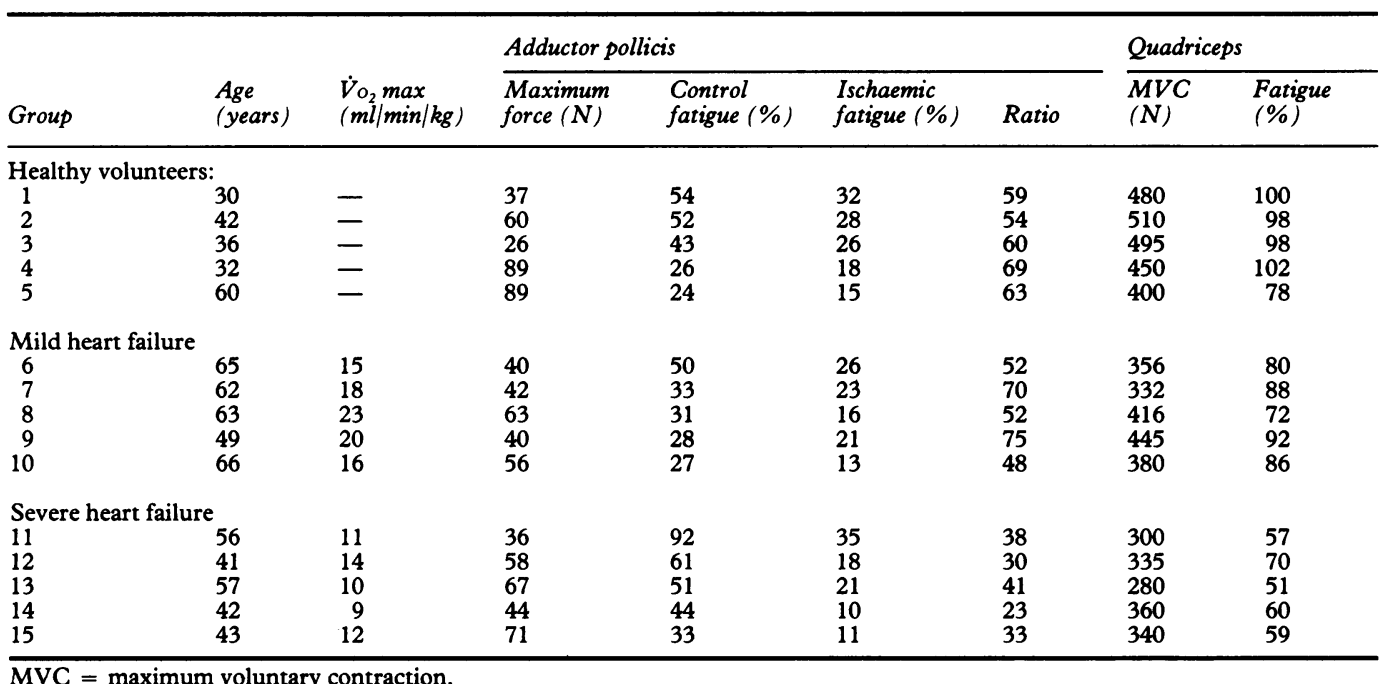

QUADRICEPS PROTOCOL

Skeletal muscle function was measured in the late morning, before lunch. The subject was seated within a rigid framework with the lower limbs hanging freely (fig 1). The right ankle was attached by an inelastic strap to a force transducer; the strap was adjusted so that it was perpendicular to the ankle. The subject wore a seat belt around the waist to prevent forward movement during quadriceps contractions. Two large saline pad electrodes were placed on the anterior aspect of the right thigh for direct transcutaneous stimulation of quadriceps muscle fibres. These electrodes were held in position by a crepe bandage. After amplification the output from the force transducer was displayed on an oscilloscope to provide visual feedback to the subject and simultaneously recorded on a UV chart recorder for subsequent analysis. The stimulating electrodes were connected to a Devices stimulator which was set to deliver a pulse duration of $1 \mathrm{~ms}$ at a frequency of $1 \mathrm{~Hz}$. After an intial period during which the subject was familiarised with the visual feedback of force production, the output voltage of the
(A)

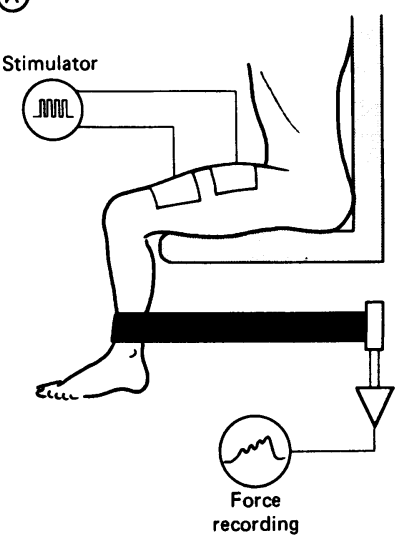

(B)

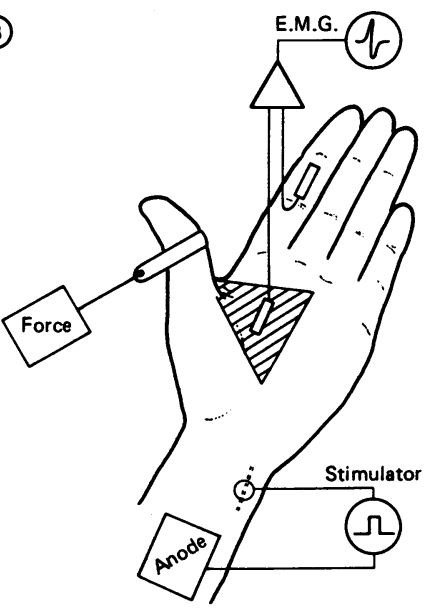

Figure 1 Experimental procedures used to measure mechanical function of the quadriceps $(A)$ and adductor pollicis $(B)$ (see text for details). EMG, electromyogram. stimulator was slowly increased to produce a muscle twitch of at least $20 \mathrm{~N}$. The subject was then instructed to pull as hard as possible on the ankle strap for about three seconds. The voluntary contraction was accepted as maximal only if the superimposed $1 \mathrm{~Hz}$ muscle twitch was abolished during the plateau of force production ${ }^{8}$ (fig 2). After maximum activation the subject rested for one minute, and then performed two further maximal contractions. The mean of the three maximum voluntary contractions was obtained by direct measurement from the UV chart recorder. The subject then rested for five minutes without muscle stimulation.

For the quadriceps fatigue protocol we set a visual target equal to $30 \%$ of the individual's measured maximum voluntary contraction. The subject then performed one second contractions followed by one second relaxations for 40 seconds. A metronome was used to facilitate coordination. This sequence was repeated each minute for 20 minutes. Maximum voluntary contraction was measured again at the end of the fatigue protocol. Fatigue was measured as the maximal voluntary force production at the end of the protocol expressed as a percentage of the initial maximal voluntary contraction.

At the end of the fatigue protocol the five patients with severe chronic heart failure had the cross sectional area of the quadriceps measured on computed tomography scans taken at mid-femur level by the method of Jones et al. ${ }^{9}$ In addition, a needle biopsy from the quadriceps was obtained from five patients (three with severe chronic heart failure and two with mild chronic heart failure) by the suction technique described by Edwards et al. ${ }^{10}$ Some of the muscle specimen was taken for histology and histochemical examination and the remainder was used to measure mitochondrial enzyme activities.

ADDUCTOR POLLICIS PROTOCOL

The subject sat at a bench and the left hand was immobilised in a padded Perspex cast. A 
Figure 2 Twitch interpolation technique used to ensure full quadriceps activation during measurement of maximum voluntary contraction.

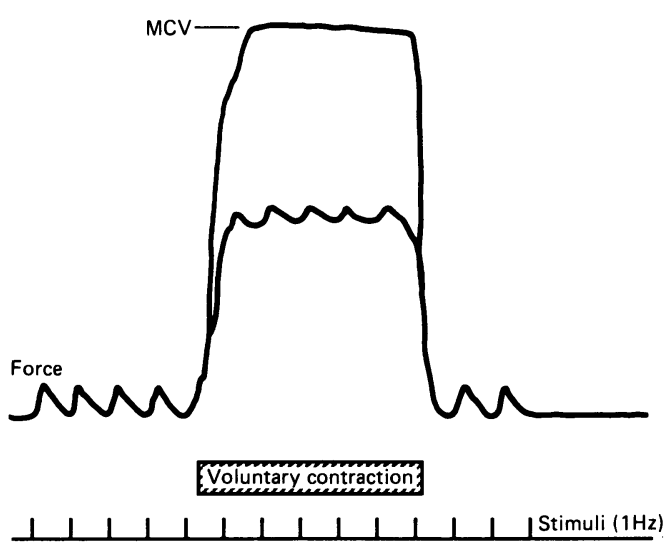

stirrup was placed over the thumb at the level of the interphalangeal joint, and connected by means of an inelastic link to a force transducer (fig 1). Surface electromyogram electrodes were taped over the belly of the adductor pollicis and on the index finger. A stimulating button electrode was placed over the ulnar nerve at the wrist, and held in position by means of an elastic strap. The indifferent electrode for the stimulator was placed high on the forearm and held in position by sticky tape. Supramaximal ulnar nerve stimulation was ensured by slowly increasing the stimulus voltage until both the surface electromyogram and isometric force had reached a plateau. The stimulus voltage for all subsequent testing was set at $10-20 \%$ above this value. The stimulus pulse width was $50 \mu \mathrm{s}$. Fatigue of the adductor pollicis was measured by recording the decline in isometric force during repetitive supramaximal tetanic ulnar nerve stimulation. The stimulation protocol consisted of intermittent once per second tetanic stimulation at $40 \mathrm{~Hz}$ for $250 \mathrm{~ms}$. During the fatigue protocol the subject was instructed to keep the left hand and forearm as relaxed as possible.

Adductor pollicis fatigue was measured twice in each subject, with a 20 minute rest between measurements. The first was performed with the circulation intact (control fatigue). The second was performed with the circulation occluded by a sphygmomanometer cuff inflated around the upper arm to a value above systolic blood pressure (ischaemic fatigue). Output from the force transducer and from the surface electromyogram was displayed on an oscilloscope, and recorded on a UV chart recorder. The maximum tetanic force of the adductor pollicis was measured as the initial force production at the beginning of the first fatigue protocol. Fatigue was measured as the force production after two minutes of intermittent tetanic stimulation expressed as a percentage of the starting value.

\section{STATISTICAL ANALYSIS}

The three groups were compared by analysis of variance. If a significant variation was indicated by the $\mathrm{F}$ distribution, further analysis was undertaken with Duncan's test and by the non-parametric Mann-Whitney test. The correlation between quadriceps maximum voluntary contraction and $\dot{\mathrm{Vo}}_{2} \max$ was assessed by standard linear regression analysis.

\section{Results}

All experiments were performed without complication. The quadriceps fatigue protocol was well tolerated. During the adductor pollicis fatigue protocol most subjects found that the ulnar stimulation was uncomfortable but tolerable.

\section{QUADRICEPS: MUSCULAR CONTRACTION}

The table shows the results for quadriceps maximum voluntary contraction and fatigue. There was a gradient in the strength of maximum voluntary contraction with the degree of heart failure in the three groups (normal $v$ mild chronic heart failure $(\mathrm{p}<0.04)$, normal $v$ severe chronic heart failure $(p<0.01)$, and mild chronic heart failure $v$ severe chronic heart failure $(p<0.09)$. Figure 3 shows the correlation between maximum voluntary contraction and $\dot{\mathrm{V}}_{2} \max$ for the 10 individual patients $(r=0.86, p<0.01)$

\section{QUADRICEPS: FATIGUE}

Fatigue in the quadriceps (table) also showed changes relating to the severity of heart failure between the three groups (normal $v$ mild chronic heart failure $(\mathrm{p}<0.09)$, normal $v$ severe chronic heart failure $(p<0.01)$, and mild chronic heart failure $v$ severe chronic heart failure $(p<0.01)$. Fatigue in quadriceps correlated with $\dot{\mathrm{Vo}}_{2} \max$ for the 10 patients with heart failure $(\mathrm{r}=0.74, \mathrm{p}<0.02)$.

QUADRICEPS: TOMOGRAPHY AND HISTOLOGICAL APPEARANCE OF BIOPSY SPECIMENS

The cross sectional area of the quadriceps in the five patients with severe chronic heart failure ranged from $44.7 \mathrm{~cm}^{2}$ to $53.7 \mathrm{~cm}^{2}$ (mean $48.2 \mathrm{~cm}^{2}$ ). The mean maximum voluntary contraction of quadriceps for the group was $323 \mathrm{~N}$, so the mean force production per unit cross sectional area was $6 \cdot 7 \mathrm{~N} / \mathrm{cm}^{2}$.

The histological and histochemical examinations showed no consistent abnormality. In two of the subjects with severe chronic heart failure type II fibre atrophy was prominent.

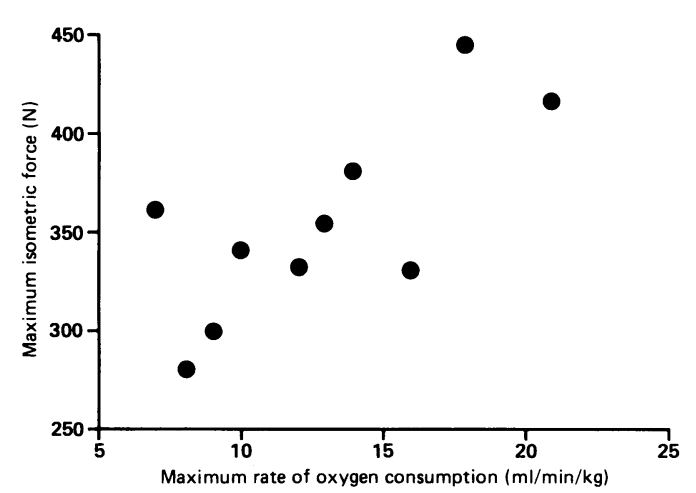

Figure 3 Relation between quadriceps maximum isometric force and maximum rate of oxygen consumption ( $\mathrm{VO}_{2}$ max) measured during symptom limited treadmill exercise test. 
ADDUCTOR POLLICIS: MUSCULAR CONTRACTION In contrast with the findings for quadriceps there was no significant difference between the maximum isometric force of adductor pollicis in the three groups (table). There was considerable variability between individuals.

\section{ADDUCTOR POLLICIS: FATIGUE}

Measurement of fatigue before circulatory occlusion (control fatigue) did not show any significant difference between the three groups. Considerable individual variation in fatigue was apparent. The lowest value for fatigue was found in a patient with severe chronic heart failure. During circulatory occlusion (ischaemic fatigue) all subjects showed a considerable increase in fatigue. There were no statistically significant differences between any of the three groups. Nevertheless, the relative increase in fatigue was significantly greater for those patients with severe chronic heart failure as judged by the ratio of ischaemic to control fatigue (table). The five patients with severe chronic heart failure showed a relatively greater increase in fatigue during circulatory occlusion than the healthy volunteers $(p<0.01)$ and patients with mild chronic heart failure $(\mathrm{p}<0.01)$.

\section{Discussion}

Previous work from our laboratory showed significant reduction of muscle strength in the quadriceps of patients with chronic heart failure, which suggested that the reduction of strength may be an important determinant of the reduced exercise tolerance. ${ }^{11}$ In the present study we confirmed these findings and showed a correlation between the maximum isometric force of quadriceps and the severity of chronic heart failure assessed by the maximum oxygen consumption on exercise.

In the group of five patients with severe chronic heart failure the maximal isometric force production per unit muscle cross sectional area was $6.7 \mathrm{~N} / \mathrm{cm}^{2}$ which is within the normal range reported by Chapman et al. ${ }^{12}$ So our results suggest that the reduction of force production seen in the large locomotive muscles of patients with chronic heart failure is due to muscle atrophy and not to impaired force generation by the myofibrils. The cause of the atrophy is unknown but may relate to disuse, repetitive ischaemia linked to reduced blood flow on exercise, or to neural and hormonal factors activated in heart failure.

Histological examination was undertaken in only five patients. The findings were identical with those we reported in a larger study. ${ }^{11} \mathrm{~A}$ wide variety of abnormalities was detected ${ }^{11}$ but there was no consistent pattern typical of heart failure. Nor were the findings typical for either rest atrophy or ischaemic induced damage. Findings were similar in the small number of biopsy specimens in the present study. All the patients in the present study had ischaemic heart disease as the cause of heart failure so that histological changes could not be attributed to an undetected myopathy which might be present in patients with idiopathic cardiomyopathy.

A further consideration is the age of the patients. The group of patients with mild heart failure was significantly older than the healthy volunteers (mean difference 21 years $(p<0.03))$ and the patients with severe heart failure (mean difference 13 years $(p<0.04)$ ). This difference could be of some importance in interpreting the results but is unlikely to be of major significance because there was no significant age difference between the healthy volunteers and patients with severe heart failure (mean difference 8 years). Furthermore, the findings in the patients with mild heart failure were, in general, intermediate between those of the healthy volunteers and the patients with severe heart failure.

In contrast with the findings in the quadriceps, maximum isometric force production of the adductor pollicis revealed no significant difference between healthy volunteers, patients with mild chronic heart failure, and patients with severe chronic heart failure. A possible explanation for this difference between the two muscles is that the use of intrinsic hand muscles (especially in the non-dominant hand) was normal in all three groups. That is to say, patients with chronic heart failure continue to use their hands for everyday activities. In contrast, the limited exercise capacity of patients with chronic heart failure results in disuse atrophy of the large locomotive muscles that contributes to the observed force reduction of the quadriceps.

In physiological terms, muscle fatigue may be defined as an inability to maintain a required level of force production. Though this definition allows for the objective measurement of muscle fatigue, such a measurement is not necessarily synonymous with the symptom of "fatigue" that is so commonly reported by patients with chronic heart failure. Nevertheless, the early onset of muscle fatigue is probably at least a marker of the processes that give rise to the perception of the symptoms of "fatigue". In the present study we showed that objective measurement of quadriceps fatigue correlates with the severity of chronic heart failure as reflected by conventional measurements of maximal exercise capacity (table). In the present studies the level of force production that we used for the quadriceps fatigue protocol was normalised to the invididual's maximum voluntary contraction. This aspect of our method is important because it made the measurement of fatigue independent of the reduced force development attributable to atrophy. In everyday activities and most functional tests-for example treadmill or bicycle exercise-an absolute level of workload and, therefore, force production is required. In this situation both reduced force production and fatigue will contribute to the subject's impaired exercise capacity.

In contrast with the findings in the quadriceps, the measurement of fatigue in the adductor pollicis under control conditions showed no significant difference between healthy volunteers and patients with mild or severe chronic 
heart failure. Small muscles might respond differently to the altered neurohormonal control of blood flow in heart failure or be less susceptible to changes in the microvasculature. Perhaps the most probable explanation is related to blood flow. Activation of a large muscle mass results in a greatly reduced skeletal muscle blood flow in patients with chronic heart failure. ${ }^{4}$ This reduced skeletal muscle blood flow is a major factor responsible for the observed increase in fatigue of the quadriceps in patients with chronic heart failure. In contrast, the adductor pollicis is a small intrinsic hand muscle and even during maximal activation the required increase in blood flow is a small proportion of the cardiac output even when chronic heart failure is severe. The last hypothesis does not accord with the idea that in severe chronic heart failure there is a generalised intrinsic abnormality of the microcirculation with skeletal muscle, such that normal vasodilatation cannot occur. ${ }^{13}$

The effect of circulatory occlusion on the development of fatigue in the adductor pollicis is more difficult to interpret. During the measurement of fatigue in the adductor pollicis under control conditions there were significant differences between the three groups. After circulatory occlusion all subjects showed a considerable increase in fatigue (as expected) but there was still no statistically significant difference among the three groups. The relative increase in fatigue after circulatory occlusion, however, was significantly greater in the patients with severe chronic heart failure. This relative increase in fatigue may be partly due to a degree of fatigue resistance under control conditions and partly a tendency towards increased fatigability after circulatory occlusion in the patients with severe chronic heart failure. An explanation for the relative increase in fatigue in the adductor pollicis after circulatory occlusion in patients with severe chronic heart failure is that there is an intrinsic alteration in skeletal muscle function which has previously been suggested in studies with magnetic resonance spectroscopy. ${ }^{67} \mathrm{Within}$ the skeletal muscle fibres of the adductor pollicis in patients with severe chronic heart failure there could be a relative shift from glycolytic to oxidative metabolic pathways for energy production combined with a reduction in myosin ATPase activity, such that the muscle fibres become relatively fatigue resistant.

The present study shows that patients with chronic heart failure show both atrophy and weakness of the large locomotive muscles. In addition, these muscles show increased fatigue, the magnitude of which correlates with the severity of chronic heart failure. The mechanism of the increased fatigue in the large locomotive muscles is possibly related to an absolute reduction in skeletal muscle blood flow. The results from the adductor pollicis suggest that muscle weakness is not an inevitable consequence of chronic heart failure, but instead supports the concept that disuse atrophy is responsible for the observed weakness of the quadriceps. The similar values for fatigue of the adductor pollicis in the three groups suggest that skeletal muscle blood flow can increase normally as long as the activated muscle mass is small. The observation of a relatively greater increase of fatigue of the adductor pollicis during circulatory occlusion in patients with severe chronic heart failure supports the hypothesis that there is an intrinsic alteration in skeletal muscle metabolism in these patients.

1 Wilson JR, Ferraro N. Exercise intolerance in patients with chronic left heart failure: relation to oxygen transport an ventilatory abnormalities. Am J Cardiol 1983;51:1358-63.

2 Saltin B. Hemodynamic adaptation to exercise. Am J Cardio 1985;55:42D-7D.

3 Wilson JR, Martin JL, Schwartz D, Ferraro N. Exercise intolerance in patients with chronic heart failure: role of impaired nutritive flow of skeletal muscle. Circulation 1984;69:1079-87.

4. Wilson JR, Martin JL, Ferraro N. Impaired skeletal muscle nutritive flow during exercise in patients with congestive nutritive flow during exercise in patients with congestive mined by the effect of dobutamine. Am J Cardiol mined by the

5 Wilson DF, Evecinska M, Drown C, Silver IA. The oxygen dependence of cellular energy metabolism. Arch Biochem Biophys 1979;195:485-94.

6 Wilson JR, Fink L, Mavis J, et al. Evaluation of energy metabolism in skeletal muscle of patients with heart failure. With gated phosphorus-31 nuclear magnetic resonance. Circulation 1985;71:57-62.

7 Wiener DH, Fink LI, Mavis J, Jones RA, Chance B, Wilson JR. Abnormal skeletal muscle bioenergetics during exercise in patients with heart failure: role of reduced muscle blood flow. Circulation 1986;73:1127-36.

8 Chapman SJ, Edwards RHT, Greig C, Rutherford O. Practical application of the twitch interpolation technique fror the study of voluntary contraction of the quadriceps for the study of voluntary contraction of the quadriceps

in man [Abstract]. J Physiol (Lond) 1984;353:3P.

Jones DA, Round JM, Edwards RHT, Grindrod SR, Toft PS. Size and composition of the calf and quadriceps muscles in Duchenne muscular dystrophy. $J$ Neurol $S$ 1983;60:307-22.

10 Edwards RHT, Round JM, Jones DA. Needle biopsy of skeletal muscle: a review of 10 years experience. Muscle Nerve 1983;6:676-83.

11 Lipkin D, Jones D, Round J, Poole-Wilson P. Abnormalities of skeletal muscle in patients with chronic heart failure. Int $J$ Cardiol 1988;18:187-95.

2 Chapman SJ Grindrod SR, Jones DA. Cross-sectional are and force production of the quadriceps muscle [Abstract] J Physiol (Lond) 1984;353:53P.

13 Zelis R, Flaim SF. Alterations in vasomotor tone in congestive heart failure. Prog Cardiovasc Dis 1982;XXIV:437-59. 